\title{
Is anti-Mullerian hormone an indicator of potential polycystic ovary syndrome in prepubertal girls with simple obesity?
}

\author{
Özlem Korkmaz, Damla Gökşen, Samim Özen, Şükran Darcan \\ Division of Pediatric Endocrinology, Department of Pediatrics, Ege University Faculty of Medicine, Izmir, Turkey. \\ E-mail: ozlem-korkmazz@hotmail.com \\ Received: 21st March 2016, Revised: 31st May 2016, Accepted: 27th October 2016
}

\begin{abstract}
SUMMARY: Korkmaz Ö, Gökşen D, Özen S, Darcan Ş. Is anti-Mullerian hormone an indicator of potential polycystic ovary syndrome in prepubertal girls with simple obesity? Turk J Pediatr 2016; 58: 406-412.

The aim of this anti-Mullerian hormone (AMH) levels in prepubertal obese girls are a predictive marker for polycystic ovary syndrome (PCOS) and to investigate the relationship between insulin resistance and AMH. Sixty girls with premature pubarche or obesity and 20 healthy controls between the ages of 6-9 were enrolled.
\end{abstract}

Of the patients, $22(36.7 \%)$ were in the obese group (Group 1), $28(46.7 \%)$ in the early pubarche group (Group 2 ) and $10(16.6 \%$ ) in the early pubarche + obese group (Group 3). Comparison of the subjects' fasting insulin and homeostatic model assessment insulin resistance (HOMA-IR) demonstrated significantly higher values for group 1 compared to group $2(p=0.001$ and 0.001 ) and, likewise, significantly higher values for group 3 compared to group 2. There was no significant differences between all groups for AMH levels.

AMH levels were not significantly different in the obese girls compared to the other groups. There was also no relationship between AMH and insulin resistance in any of the groups. Further studies, however, are needed due the limited number of subjects in this study and in the absence of adequate relevant data.

Key words: antimullerian hormone, polycystic ovary syndrome, obesity, early pubarche

The anti-mullerian hormone $(\mathrm{AMH})$ is a peptide growth factor from the transforming growth factor-Beta (TGF-Beta) super-family. It is expressed from preantral and small antral follicle granulosa cells and plays a mediator role particularly in primordial follicle development and follicle selections. Cessation of $\mathrm{AMH}$ expression from the follicles is necessary for dominant follicle formation ${ }^{1,2}$. Serum AMH levels diminish with age, although the decline has been shown to be slower in patients with Polycystic ovary syndrome (PCOS). PCOS is an endocrine disorder characterized by irregular menstrual cycles with clinical and laboratory manifestations of hyperandrogenis ${ }^{3}$. The syndrome is characterized by increased preantral follicle count. Women with PCOS were shown to have higher $\mathrm{AMH}$ levels compared to healthy women. Different studies have also reported higher AMH levels in prepubertal and peripubertal daughters of women with PCOS ${ }^{4-6}$. These reports suggest that changes in $\mathrm{AMH}$ production associated with PCOS may be observed even during the prepubertal period. The exact cause of the increased $\mathrm{AMH}$ production in patients with PCOS is not known ${ }^{4,7}$.

Early pubarche develops as a result of premature production and release of adrenal androgens ${ }^{8}$. The hyperinsulinism found in the girls with early pubarche has been asserted as a triggered factor in adrenal androgens and ovarian hypersecretion and so in the development of PCOS.

There are a few studies investigating the relationship between AMH and obesity. Obese girls are at a higher risk for $\mathrm{PCOS}^{9}$. As of the date the present study was planned, there were no studies in the literature investigating the relationship between AMH and PCOS 
in prepubertal girls with simple obesity. Timely diagnosis of PCOS will ensure early identification of long-term complications and help predicting possible risks. The present study was designed to determine whether the $\mathrm{AMH}$ may be an early indicator of a potential polycystic ovarian syndrome in prepubertal girls with simple obesity, and to establish any relationship between insulin resistance and the $\mathrm{AMH}$.

\section{Material and Methods}

Girls who had presented to the Pediatric Endocrinology Department Ege University Faculty of Medicine for early pubarche or obesity whose first examination data were available, who were still under follow up and whose pubarche manifestations had developed before the age of 8 were included as the study group. Since prepubertal individuals were to be assessed, patients younger than 9 years were included. Those whose early pubarche was associated with sex hormone releasing tumors, those diagnosed with congenital adrenal hyperplasia, those whose obesity was associated with the Cushing's Syndrome and those with syndromic obesity were also excluded. Further exclusion criteria were presence of concomitant diabetes mellitus (DM), thyroid dysfunction or hyperprolactinemia. Sixty girls meeting these criteria were included. Patients were divided into 3 groups: Group 1: obese group (no early pubarche), Group 2: early pubarche group (nonobese), Group 3: early pubarche+obese group. As controls, 20 non-obese girls with no known chronic conditions who presented to the
Pediatrics outpatient clinic of were included. The patient and control groups included girls only. All patients were pre-pubertal. Information registered at the time of first presentation (birth weight, age at presentation, age at onset of axillary and/or pubic pilosity, auxological data at presentation, bone age, acne presence) were obtained from the patient files. Left wrist graph and Greulich-Pyle scale were used for bone age assessments ${ }^{10}$. Consents to participate in the study were obtained from the subjects and/or their parents (Informed Subject Consent Form). Familial history, medical history and medication history were examined. Each subject underwent a detailed clinical examination by the same investigator, and pubertal development was assessed as per Tanner staging ${ }^{11}$. Body weight measurements were performed with "Seca (Ulmer)" scale and height measurements with "BUSSE Design Engineering ulmer stadiometer". Weight and height SDs, Body Mass Indices (BMI) and BMI SDs were calculated based on the standards described for Turkish children ${ }^{12}$. Body fat was measured by "TANITA Corporation - Quality Control Seal" using bioelectrical impedance method. Body fat volume, lean body mass, fat/muscle ratio and body water ratio values were obtained. The subjects were weighed with bare feet and with light clothes on, and the measurements were performed in supine position. All subjects in the patient group were studied for Follicle stimulating hormone (FSH), Luteinizing hormone ( $\mathrm{LH})$, estradiol, free and total testosterone, dehydroepiandrosteronesulfate (DHEA-S), $17 \mathrm{OH}$ Progesterone, free throxine (fT4), thyroid-stimulating hormone

Table I. Mean Values for Subjects' Demographics and Corresponding Standard Deviations

\begin{tabular}{|c|c|c|c|c|}
\hline & $\begin{array}{c}\text { Group } 1 \\
\mathrm{n}: 22(36.7 \%)\end{array}$ & $\begin{array}{c}\text { Group } 2 \\
\mathrm{n}: 28(46.7 \%)\end{array}$ & $\begin{array}{c}\text { Group } 3 \\
\mathrm{n}: 10(16.6 \%)\end{array}$ & $\begin{array}{c}\text { Control } \\
\text { n: } 20\end{array}$ \\
\hline Age & $7.69 \pm 0.90$ & $7.62 \pm 0.77$ & $8.20 \pm 0.89$ & $7.42 \pm 0.88$ \\
\hline Body weight $\mathrm{SD}^{*}$ & $4.14 \pm 1.27$ & $0.94 \pm 0.94$ & $3.16 \pm 1.20$ & $0.01 \pm 1.12$ \\
\hline Height SD & $1.21 \pm 1.08$ & $0.80 \pm 1.05$ & $1.49 \pm 1.24$ & $0.34 \pm 1.20$ \\
\hline BMI SDS** & $2.91 \pm 0.46$ & $0.64 \pm 0.80$ & $2.61 \pm 0.40$ & $-0.40 \pm 1.22$ \\
\hline Birth weight SD & $-0.15 \pm 0.81$ & $-0.19 \pm 1.16$ & $-0.49 \pm 1.13$ & $-0.32 \pm 1.11$ \\
\hline \multicolumn{3}{|c|}{$\begin{array}{l}* p<0.05 \text { between the group } 1 \text { and group } 2 \\
\text { between the group } 1 \text { and control group } \\
\text { between the group } 2 \text { and group } 3 \\
\text { between the group } 3 \text { and control group }\end{array}$} & \multicolumn{2}{|c|}{$\begin{array}{l}\text { between the group } 1 \text { and control group } \\
\text { between the group } 1 \text { and group } 2 \\
\text { between the group } 2 \text { and group } 3 \\
\text { between the group } 3 \text { and control group } \\
\text { between the group } 2 \text { and control group }\end{array}$} \\
\hline
\end{tabular}

SD: Standart Deviation; BMI: Body Mass Index; SDS: Standart Deviation Score 
Table II. Adrenal Axis Assessment

\begin{tabular}{lccc}
\hline & Group 1 & Group 2 & Group 3 \\
\hline ACTH $(\mathrm{pg} / \mathrm{ml})$ & $33.1 \pm 18.1$ & $32.3 \pm 14.8$ & $41.0 \pm 17.5$ \\
Cortisole $(\mu \mathrm{g} / \mathrm{dl})$ & $9.92 \pm 4.10$ & $12.2 \pm 4.00$ & $12.5 \pm 4.35$ \\
fTestosterone* $(\mathrm{pg} / \mathrm{ml})$ & $1.04 \pm 0.58$ & $1.06 \pm 0.41$ & $1.72 \pm 0.79$ \\
tTestosterone $(\mathrm{ng} / \mathrm{dl})$ & $0.10 \pm 0.09$ & $0.13 \pm 0.08$ & $0.12 \pm 0.14$ \\
Androstenedione $(\mathrm{ng} / \mathrm{ml})$ & $0.80 \pm 0.82$ & $0.83 \pm 0.43$ & $1.99 \pm 2.06$ \\
17-OH Progesterone $(\mathrm{ng} / \mathrm{ml})$ & $0.81 \pm 0.83$ & $0.96 \pm 0.50$ & $0.85 \pm 0.37$ \\
DHEA-S** $(\mu \mathrm{g} / \mathrm{dl})$ & $51.3 \pm 37.4$ & $84.8 \pm 43.1$ & $92.3 \pm 61.9$ \\
\hline
\end{tabular}

${ }^{*} p<0.05 \quad$ between group 1 and group $3{ }^{* *} p<0.05$ between group 1 and group 3

between group 2 and group $3 \quad$ between group 1 and group 2

ACTH: Adrenocorticotropic Hormone, DHES-A: Dehydroepiandrosterone Sulfate

(TSH), prolactin, androstenedione, fasting blood glucose, fasting insulin, fasting lipid, adrenocorticotropic hormone (ACTH), cortisol and $\mathrm{AMH}$ levels. Subjects included as controls were studied for AMH, FSH, LH, estradiol, free and total testosterone, DHEA-S, free T4, TSH and prolactin. Insulin analyses were performed using the Immulite ${ }^{\circledR} 2000$ - Siemens device with the enzyme labeling method. FSH, LH, estradiol, total testosterone, DHEA-S, free T4, $\mathrm{TSH}$, prolactin were studied with the Modular Analytics - E170 Roche device using the enzyme labeling method while Free testosterone and $17 \mathrm{OH}$ progesterone were studied with the RIA method. Androstenedione and ACTH were measured using Immulite/Immulite 1000 with the immunoassay method. AMH was studied with the Immunotech/Beckman Coulter instrument using the Elisa method. HOMA-IR calculations used the following formula: Fasting glucose (FG; mmol/l) $\mathrm{x}$ fasting insulin (FI; $\mathrm{mcU} / \mathrm{ml}) / 22.5^{13}$.

\section{Statistical analysis}

According to the number of samples compatibility of data to the normal distribution was tested by the Shapiro-Wilk test. $P>0.05$ was accepted in accordance with the normal distribution. Inter-group differences were assessed by the one-way variance analysis in cases fit to normal distribution while KruskalWallis test was used in cases not fitting to normal distribution. Unless otherwise specified, all data were expressed as mean \pm standard deviation. The data were analyzed using the SPSS 17.0 statistical software (IBM SPSS Statistics $17 \mathrm{R})$. Statistical significance was set at $p<0.05$.

\section{Results}

Of the patients, $22(36.7 \%)$ were in the obese group (Group 1), 28 (46.7\%) in the early pubarche group (Group 2) and 10 (16.6\%) in the early pubarche + obese group (Group 3 ).

When the subjects were grouped by obesity and pubarche state, age distribution was comparable among the four groups. The four groups did not differ significantly regarding height, height SD, birth weight and birth weight SD $(p>0.05)$ (Table I).

According to the pubic pilosity assessment of subjects who presented with group 2, 26 $(92.9 \%)$ of the subjects were stage 2 and 2 $(7.1 \%)$ were stage 3 . Pubic pilosity assessment of subjects with group 3 demonstrated that $8(80 \%)$ of the subjects were stage 2 and 2 (20\%) were stage 3 .

Comparison of group 1, group 2 and group 3 in terms of laboratory parameters yielded comparable FSH, LH, LH/FSH, total testosterone, 17-OH-Progesterone, androstenedione, fT4, $\mathrm{TSH}, \mathrm{ACTH}$, cortisole and estradiol levels $(p>0.05)$ (Table II-III).

Comparison of group 1, group 2, group 3 and control group did not demonstrate a significant difference in AMH levels between the four groups $(p=0.987)$ (Table III).

In our study, comparison of prolactin and free testosterone levels demonstrated that group 3 had higher prolactin and free testosterone levels compared to group 1 and group $2(p<0.05)$. Group 2 and group 3 had higher DHEA-S levels compared to group $1(p=0.002$ and 0.023$)$.

Comparison of AMH levels among 29 subjects 
Table III. Comparison of Sex Hormone and AMH Levels

\begin{tabular}{lcccc}
\hline & Group 1 & Group 2 & Group 3 & Control \\
\hline FSH $(\mathrm{mIU} / \mathrm{ml})$ & $2.04 \pm 1.63$ & $2.24 \pm 1.42$ & $2.40 \pm 2.47$ & NA \\
LH $(\mathrm{mIU} / \mathrm{ml})$ & $0.27 \pm 0.17$ & $0.37 \pm 0.52$ & $0.44 \pm 0.49$ & NA \\
LH/FSH & $0.18 \pm 0.15$ & $0.15 \pm 0.17$ & $0.29 \pm 0.37$ & NA \\
Prolactin $(\mathrm{ng} / \mathrm{ml})$ & $8.66 \pm 3.68$ & $8.20 \pm 4.14$ & $13.3 \pm 7.02$ & NA \\
Estradiol $(\mathrm{ng} / \mathrm{ml})$ & $22.0 \pm 9.26$ & $19.3 \pm 2.54$ & $18.5 \pm 4.74$ & NA \\
AMH $(\mathrm{ng} / \mathrm{ml})$ & $1.97 \pm 1.36$ & $2.24 \pm 1.79$ & $2.24 \pm 1.99$ & $2.05 \pm 1.50$ \\
\hline
\end{tabular}

FSH: Follicle Stimulating Hormone; LH: Luteinizing Hormone; AMH: Anti-Mullerian Hormone

with high DHEA-S levels yielded a significant difference between the obese subjects and early pubarche subjects. Among the cases whose DHEA-S levels were high, the AMH levels were higher in early pubarche groups. $(p=0.033)$.

The subjects did not differ regarding bone ages $(p>0.05)$.

Comparison of the subjects' fasting insulin and HOMA-IR levels demonstrated statistically higher levels of insulin and HOMA-IR for group 1 compared to group $2(p=0.001$ and 0.001$)$ and significantly higher insulin and HOMA-IR levels in group 3 versus group $2(p=0.014$ and 0.006) (Table IV).

In the group 1, AMH levels were negatively correlated with free testosterone $(p=0.004$, $\mathrm{R}=-0.598)$, DHEA-S $(p=0.003, \mathrm{R}=-0.608)$ and $17-\mathrm{OH}$ progesterone $(p=0.021, \mathrm{R}=-0.500)$ levels. No correlations were identified between $\mathrm{AMH}$ and BMI, BMI SD, body fat percentage, $\mathrm{LH}, \mathrm{FSH}, \mathrm{LH} / \mathrm{FSH}$, androstenedione and insulin levels.

In group 2 , there was a positive correlation between AMH and bone age $(p=0.006$, $\mathrm{R}=0.507)$ and androstenedione levels $(\mathrm{p}=0.037, \mathrm{R}=0.420)$. AMH and BMI, BMI $\mathrm{SD}$, body fat percentage, LH, FSH, LH/FSH, free testosterone, total testosterone, DHEA-S, 17-OH Progesterone or insulin levels were not correlated.

In group 3, there was a negative correlation between the AMH level and BMI SD $(p=0.045$, $\mathrm{R}=-0.678$ ). No correlations between $\mathrm{AMH}$ and body fat percentage, LH, FSH, LH/FSH, free testosterone, total testosterone, DHEA-S, 17$\mathrm{OH}$ Progesterone, androstenedione or insulin levels were determined.

\section{Discussion}

Alternations in gonadotropin release, steroid synthesis defects, impaired insulin release and activity accompanied by genetic factors are prominent features in the pathophysiology of PCOS. Insulin resistance appears to play an important role in this mechanism and is present in $50-70 \%$ of the cases, independent of obesity ${ }^{14,15}$. Insulin resistance and resultant insulin over-expression leads to increased androgen formation. Insulin resistance was not observed in any of the patients who presented with pubarche in this study. Early pubarche develops as a result of premature production and release of adrenal androgens, and may be an indicator of PCOS ${ }^{8}$. Based on the hypothesis that AMH may be an indicator of a potential PCOS in individuals with early pubarche, a study on prepubertal girls with early adrenarche found lower serum AMH levels in this population compared to the controls, not supporting this hypothesis 4 . In our study, the subjects in group 2 and the control group did not differ significantly and the subgroup analysis did not result in any relevant differences between group 1, group 2 , group 3 and control group regarding the AMH levels.

Serum AMH levels are higher in women with hyperandrogenism and PCOS than in women with PCOS with normal levels of androgen ${ }^{16}$. In a study conducted by Oncul $\mathrm{M}$, et al., ${ }^{17}$ it was shown that serum AMH levels were detected to be higher in patients with PCOS (including polycystic ovarian morphology, oligoanovulation and hyperandrogenemia criteria) compared to the patients with late-onset congenital adrenal hyperplasia. According to the authors, these findings suggest that serum $\mathrm{AMH}$ level could be a marker in differential 
Table IV. Comparison of Metabolic Parameters

\begin{tabular}{lccc}
\hline & Group 1 & Group 2 & Group 3 \\
\hline Fasting blood glucose $(\mathrm{mg} / \mathrm{dl})$ & $86.6 \pm 8.70$ & $85.9 \pm 7.30$ & $86.6 \pm 8.70$ \\
Fasting insulin* $(\mathrm{mIU} / \mathrm{ml})$ & $9.42 \pm 8.03$ & $4.13 \pm 3.59$ & $7.85 \pm 4.67$ \\
HOMA-IR* & $2.02 \pm 1.80$ & $0.82 \pm 0.76$ & $1.65 \pm 0.91$ \\
\hline
\end{tabular}

${ }^{*} p<0.05$ between group 1 and group 2

between group 3 and group 2

HOMA-IR: Homeostatic Model Assessment Insulin Rezistance

diagnosis between late-onset congenital adrenal hyperplasia and PCOS which includes three main criteria. In a study of patients with adult PCOS, free testosterone, total testosterone and DHEA-S levels were higher compared to the control group, and a positive correlation was determined between AMH levels and free and total testosterone levels in the PCOS group ${ }^{18}$. Another study of peripubertal daughters of women with PCOS, AMH and testosterone levels were distinctly higher compared to the control group 5 . In our study, free testosterone levels were higher in group 3 compared to groups 1 and 2 . Given that obesity and early pubarche are risk factors for PCOS, coexistence of these two conditions may further increase the risk of PCOS as well as the severity of resulting metabolic disorders. We believe that this is why free testosterone levels were higher in group 3 compared to other groups.

In a study evaluating AMH levels in girls with early adrenarche, DHEA-S levels were higher in this group compared to controls and there was an inverse correlation between serum AMH and DHEA-S levels in the control group, whereas no correlation was detected in patients with early adrenarche ${ }^{4}$. DHEA-S levels were also higher in group 2 and group 3 compared to the group 1 in our study. In addition, AMH and DHEA-S was not correlated in group 2 or group 3. There was, however, a negative correlation between AMH and DHEA-S in group 1. The comparison of AMH levels among a total of 29 subjects with high DHEA-S levels in all groups demonstrated a significant difference between the obese subjects and early pubarche subjects. $\mathrm{AMH}$ levels were higher in the early pubarche group. Consequently, since AMH cannot be used as a PCOS indicator in prepubertal obese girls and increased androgen levels are relevant for PCOS, it can be concluded that $\mathrm{AMH}$ might be a more relevant indicator of potential PCOS in patients with early pubarche with high DHEA-S levels. However, in the study by Rosenfield ${ }^{19}$, elevations in $\mathrm{AMH}$ and testosterone levels in children of women with PCOS were not simultaneous, with AMH and testosterone levels increasing during the late puberty. In another study investigating increased androgen levels in adolescent PCOS patients, positive relationships were identified between androgen levels, androstenedione and testosterone levels and $\mathrm{AMH}^{20}$. In our study, there was a positive correlation between AMH and androstenedione levels in group 2. This supports that AMH production is affected by androgen levels. Androgens may increase follicle count, thereby augmenting AMH production from the granulosa cells from maturing follicles. However, several extra-ovarian and intra-ovarian factors including increased LH secretion, hyperinsulinemia, peptides of oocyte origin and intra-ovarian proteins may also result in abnormal follicle growth or development. This may be more pronounced with later stages of puberty and may be affecting AMH production.

Although there are studies determining positive and negative correlations between AMH and insulin resistance in adult subjects, other studies identifying no correlation between these two parameters do exist ${ }^{21}$. In our study, comparison of subjects' fasting insulin and HOMA-IR levels demonstrated significantly higher insulin and HOMA-IR levels in group 1 compared to group 2 and control group as well as significantly higher insulin and HOMA-IR levels in group 3 compared to those of group 2. There was no significant difference between group 2 and the control group. Our study supports the association between obesity and insulin resistance during prepuberty. In our subjects, AMH was not correlated with basal insulin or HOMA-IR levels in group 1, group 2 or group 3 . We believe this supports that 
basal insulin levels measured during prepuberty may not be a satisfactory indicator for potential PCOS and therefore 2-hour insulin levels as measured by OGTT, as suggested by Crisosto $\mathrm{N}$, et al. ${ }^{5}$ should also be studied.

There are many studies which assessed the relationship between AMH and obesity in adulthood. Significantly lower levels of $\mathrm{AMH}$ have been reported in obese women in comparison with women with normal body weight. This difference has been attributed to the suppression of local AMH production due to the increased estrogen/androgen ratio and high local aromatase activity ${ }^{22}$. Another suggested mechanism involves inhibition of aromatase activity in the ovaries by adiponectins, altering $\mathrm{AMH}$ production in granulosa cells ${ }^{23}$. However, there are studies that do not support the relationships between AMH and BMI. In a study of adult women with PCOS, PCOS and control groups did not differ significantly regarding $\mathrm{BMI}$ and $\mathrm{AMH}$ assessments ${ }^{24}$. In our study, no significant difference in $\mathrm{AMH}$ levels was observed in group 1 compared to group 2, group 3 or the control group. This indicates that $\mathrm{AMH}$ levels cannot be used as an indicator of PCOS in obese prepubertal girls. In a study comparing girls with early adrenarche with a median age of 7.6 (4.88.9) with controls, a negative correlation was identified between AMH and BMI SD and body fat ratio. Lower $\mathrm{AMH}$ levels of the subjects with early adrenarche may be explained by their higher body weight and body fat percentage compared to the controls 4 . In our study, there was also a negative correlation between AMH levels and BMI SD in the group 3. However, when group 1, group 2 and the control group were evaluated separately, AMH levels and BMI were not correlated. This may be explained by the reduced AMH levels as a result of suppression of $\mathrm{AMH}$ production in follicles due to obesity in obese individuals with early pubarche, when early pubarche is considered an indicator for PCOS.

In conclusion, the AMH level which is measured in the prepubertal period of obese girls cannot be used as an early indicator for PCOS that can be developed in the future. Further longitudinal investigations are necessary to achieve accurate results. Also further studies are needed due to the limited number of subjects in this study and in the absence of adequate relevant data.

\section{Acknowledgements}

This study was supported by the Department of Research Project of Ege University.

\section{REFERENCES}

1. Pellatt L, Rice S, Mason HD. Anti-Mullerian hormone and polycystic ovary syndrome: a mountain too high? Reproduction 2010; 139: 825-833.

2. Itman C, Mendis S, Barakat B, Loveland KL. All in the family: TGF-beta family action in testis development. Reproduction 2006; 132: 233-246.

3. Norman RJ, Wu R, Stankiewicz MT. Polycystic ovary syndrome. Med J Aust 2004; 180: 132-137.

4. Utriainen P, Jaaskelainen J, Voutilainen R. Serum antimullerian hormone concentrations in prepubertal girls with and without premature adrenarche: The influence of body mass index. Horm Res Paediatr 2010; 74: 207-211.

5. Crisosto N, Codner E, Maliqueo M, et al. Anti-Mullerian hormone levels in peripubertal daughters of women with polycystic ovary syndrome. J Clin Endocrinol Metab 2007; 92: 2739-2743.

6. Siow Y, Kives S, Hertweck P, Perlman S, Fallat ME. Serum Mullerian-inhibiting substance levels in adolescent girls with normal menstrual cycles or with polycystic ovary syndrome. Fertil Steril 2005; 84: 938944.

7. La Marca A, Orvieto R, Giulini S, et al. Mullerianinhibiting substance in women with polycystic ovary syndrome: relationship with hormonal and metabolic characteristics. Fertil Steril 2004; 82: 970-972.

8. Ibanez L, Valls C, Potau N, Marcos MV, de Zegher F. Polycystic ovary syndrome after precocious pubarche: ontogeny of the low-birthweight effect. Clin Endocrinol (Oxf) 2001; 55: 667-672.

9. Yildiz BO, Knochenhauer ES, Azziz R. Impact of obesity on the risk for polycystic ovary syndrome. J Clin Endocrinol Metab 2008; 93: 162-168.

10. Greulich WW, Pyle Sl. Radiographic Atlas of Skeletal Devolepment of the Hand and Wrist. (2 ${ }^{\text {nd }}$ ed). Stanford: Stanford University Press, 1958: 125-228.

11. Tanner JM. Recording adolescent physical changes: Tanner system. Del Med J 1973; 45: 84-88.

12. Neyzi O, Binyıldız P, Alp H. Türk çocuklarında büyüme ve gelişme normları. I-tartı ve boy değerleri. İstanbul Tip Fakültesi Mecmuası, 1978; 41 (Suppl.74): 1-22.

13. Kajaia N, Binder H, Dittrich R, et al. Low sex hormonebinding globulin as a predictive marker for insulin resistance in women with hyperandrogenic syndrome. Eur J Endocrinol 2007; 157: 499-507.

14. Dunaif A. Insulin resistance and the polycystic ovary syndrome: mechanism and implications for pathogenesis. Endocr Rev 1997; 18: 774-800.

15. Legro RS, Gnatuk CL, Kunselman AR, Dunaif A. Changes in glucose tolerance over time in women with polycystic ovary syndrome: a controlled study. J Clin Endocrinol Metab 2005; 90: 3236-3242. 
16. Eldar-Geva T, Margalioth EJ, Gal M, et al. Serum antiMullerian hormone levels during controlled ovarian hyperstimulation in women with polycystic ovaries with and without hyperandrogenism. Hum Reprod 2005; 20: 1814-1819.

17. Oncul M, Sahmay S, Tuten A, et al. May AMH levels distinguish LOCAH from PCOS among hirsute women? Eur J Obstet Gynecol Reprod Biol 2014; 178: 183-187.

18. Woo HY, Kim KH, Rhee EJ, Park H, Lee MK. Differences of the association of anti-Mullerian hormone with clinical or biochemical characteristics between women with and without polycystic ovary syndrome. Endocr J 2012; 59: 781-790.

19. Rosenfield RL. Clinical review: Identifying children at risk for polycystic ovary syndrome. J Clin Endocrinol Metab 2007; 92: 787-796.

20. Park AS, Lawson MA, Chuan SS, et al. Serum anti-mullerian hormone concentrations are elevated in oligomenorrheic girls without evidence of hyperandrogenism. J Clin Endocrinol Metab 2010; 95: 1786-1792.
21. Thomson RL, Buckley JD, Moran LJ, et al. The effect of weight loss on anti-Mullerian hormone levels in overweight and obese women with polycystic ovary syndrome and reproductive impairment. Hum Reprod 2009; 24: 1976-1981.

22. Andersen CY, Byskov AG. Estradiol and regulation of anti-Mullerian hormone, inhibin- $A$, and inhibin-B secretion: analysis of small antral and preovulatory human follicles' fluid. J Clin Endocrinol Metab 2006; 91: 4064-4069.

23. Ledoux S, Campos DB, Lopes FL, et al. Adiponectin induces periovulatory changes in ovarian follicular cells. Endocrinology 2006; 147: 5178-5186.

24. Woo HY, Kim KH, Rhee EJ, Park H, Lee MK. Differences of the association of anti-Mullerian hormone with clinical or biochemical characteristics between women with and without polycystic ovary syndrome. Endocr J 2012; 59: 781-790. 